\title{
Bearing-only Target Tracking using a Bank of MAP Estimators
}

\author{
Guoquan P. Huang, Ke X. Zhou, Nikolas Trawny, and Stergios I. Roumeliotis
}

\begin{abstract}
Nonlinear estimation problems, such as bearingonly tracking, are often addressed using linearized estimators, e.g., the extended Kalman filter (EKF). These estimators generally suffer from linearization errors as well as the inability to track multimodal probability density functions (pdfs). In this paper, we propose a bank of batch maximum a posteriori (MAP) estimators as a general estimation framework that provides relinearization of the entire state history, multi-hypothesis tracking, and an efficient hypothesis generation scheme. Each estimator in the bank is initialized using a locally optimal state estimate for the current time step. Every time a new measurement becomes available, we convert the nonlinear cost function corresponding to this relaxed one-step subproblem into polynomial form, allowing to analytically and efficiently compute all stationary points. This local optimization generates highly probable hypotheses for the target trajectory and greatly improves the quality of the overall MAP estimate. Additionally, pruning and marginalization are employed to control the computational cost. Monte Carlo simulations and real-world experiments show that the proposed approach significantly outperforms the EKF, the standard batch MAP estimator, and the particle filter (PF), in terms of accuracy and consistency.
\end{abstract}

\section{INTRODUCTION}

In this paper we present a general framework to improve consistency of nonlinear estimation. We apply our method to the particular problem of bearing-only target tracking, i.e., estimating the state of a moving target using only bearing measurements from a single mobile sensor whose position and orientation are known. Bearing-only tracking has attracted significant interest over the past decades, since it arises in a variety of applications, such as submarine tracking using towed-array sonar and aircraft surveillance using radar in passive mode [1], [2]. Examples of recent research in this area include design of new estimators and adaptive control of sensor motions [3], [4].

Nonlinear estimation problems such as bearing-only tracking are often addressed using linearized estimators, e.g., the extended Kalman filter (EKF). These estimators suffer from linearization errors and the inability to track multimodal probability density functions (pdfs), which often arise in nonlinear estimation problems. Several methods have been proposed to reduce linearization errors. The iterated EKF (IEKF) [2], for example, iterates the filter update to convergence, relinearizing the measurement function at every

This work was supported by the University of Minnesota (DTC), and the National Science Foundation (IIS-0643680, IIS-0811946, IIS-0835637).

G. P. Huang and S. I. Roumeliotis are with the Department of Computer Science and Engineering, University of Minnesota, Minneapolis, MN 55455 , USA. Email: \{ghuang|stergios\}@Cs. umn . edu

K. X. Zhou is with the Department of Electrical and Computer Engineering, University of Minnesota, Minneapolis, MN 55455, USA. Email: kezhou@ece. umn.edu

N. Trawny is with the NASA Jet Propulsion Laboratory, Pasadena, CA 91109, USA. Email: nikolas.trawnye jpl.nasa.gov step. Alternatively, the unscented Kalman filter (UKF) [5] deterministically samples the nonlinear function around the estimate and thus reduces linearization errors. However, any (explicit or implicit) linearization-based filtering approach marginalizes all but the current state, and is hence unable to refine past linearization points. In contrast, a batch maximum a posteriori (MAP) estimator [6] computes the estimates for the states at all time steps using all available measurements. This allows continuous relinearization of the entire state trajectory, which greatly reduces linearization errors. However, just as the IEKF and its variants, the batch MAP estimator can only track one of the potentially many modes of the posterior pdf. Only a few estimators, such as the multihypothesis EKF (MHEKF) [3], and the particle filter (PF) [7], are specifically designed to treat multimodal distributions by simultaneously tracking a set of different state estimates. Most of the time though these hypotheses are generated randomly, wasting in effect a considerable portion of the computational resources.

The estimation framework presented in this paper provides both relinearization and multi-hypothesis tracking, together with a highly efficient hypothesis generation scheme. The ideal approach to batch MAP estimation would be to compute all modes of the posterior pdf, thus ensuring a globally optimal estimate. However, as our analysis will show, this approach is computationally intractable due to the growing size of the state vector. We therefore relax the problem, and optimize only for the current target position at each time step, treating the state history of each hypothesis as a constant prior. Using an inferred measurement, we convert the nonlinear cost function of this subproblem into polynomial form, and subsequently employ algebraic geometry techniques [8] to analytically compute all stationary points and thus all modes of the pdf. Each mode is used to initialize a new MAP estimator in the bank, thus allowing to track the most probable hypotheses of the state trajectory, and in turn greatly improving the accuracy and consistency of the MAP estimate. At the same time, we achieve low, resource-adaptive computational cost through pruning and marginalization. The former controls the exponential growth of hypotheses, whereas the latter limits the size of the state vector.

Apart from the particular application of bearing-only target tracking, the proposed framework is applicable to a broad class of nonlinear estimation problems in robotics and computer vision that can be expressed in (or converted into) polynomial form. For instance, in our recent work [9], we successfully applied this approach to significantly improve the performance of range-only target tracking. In this paper, we extend our previous work to a more challenging problem 
for which we provide a detailed analysis, as well as validation through simulation and a real-world experiment.

\section{RELATED WORK}

Bearing-only tracking has been studied for decades and many algorithms, both batch and recursive, have been proposed in the literature [2], [10]. Among these algorithms, the EKF is one of the most widely used methods. However, the inability of the EKF to relinearize previous states of the nonlinear system, when new measurements become available, often leads to large linearization errors which negatively impact its performance. This has given rise to refinements of the EKF, such as the modified-polar coordinates EKF [11] and the shifted Rayleigh filter (SRF) [4]. However, both of these EKF variants can only track a single mode of the posterior pdf of the target state and thus suffer from the same problem as the EKF, i.e., they can potentially track an incorrect mode of the posterior pdf and hence become inconsistent.

To mitigate the aforementioned issue, an MHEKF was proposed in [3] to track multiple hypotheses of the target state. The MHEKF makes an assumption about the minimum and maximum distance between the sensor and target and divides this range interval into a number of subintervals, each representing a hypothesis regarding the true range of the target. A bank of independently operating range-parameterized EKFs is then created, each designed for one of the hypotheses and receiving the same bearing measurements. The MHEKF described in [3] determines a fixed number of EKFs when the first measurement becomes available. This idea was extended in [12] so that the filter bank can dynamically change its size at each time step based on the current measurement likelihood. Since no filter in the MHEKF can guarantee computing the globally optimal estimate (due to the multimodal nature of the posterior pdf and the filter's inability to relinearize the nonlinear measurement function involving past states), this approach can also become inconsistent and diverge. Note also that this method assumes prior knowledge about the maximum target distance, which might not always be available in real applications. More importantly, this approach does not provide a measurable criterion about how many partitions are needed in the assumed range interval and where to choose them. In contrast, our proposed bank of MAP estimators selects most probable hypotheses of the target trajectory based on local optimality at each time step.

The PF [7], [13]-[15] has recently attracted considerable interest, due to its ability to address nonlinear estimation problems with multimodal pdfs. In the standard PF, each particle represents a hypothesis of the target state, weighted by its measurement likelihood. If the particles sample the state space sufficiently, the PF will converge to the true distribution. However, the particles are usually initialized randomly, and if far from a mode of the pdf, their weights will decay quickly and lead to particle depletion, even if resampling is employed. Therefore, in order to provide accurate estimates, the PF requires a large number of particles, thus resulting in substantial computational demands. In contrast, the proposed bank of MAP estimators analytically computes all modes of the posterior pdf at the current time step and efficiently focuses the computational resources on the most probable trajectory hypotheses.

\section{PROBLEM Formulation}

In this section, we formally describe the problem formulation of bearing-only tracking. Consider a single sensor that moves in a plane and estimates the kinematic state (position, velocity, etc.) of a moving target by processing noisy bearing measurements. In this work, we study the case of global tracking where the position of the target is expressed with respect to a fixed (global) frame of reference, instead of a relative sensor-centered one. We hereafter assume known sensor pose, as well as known parameters of the target stochastic motion model. The state vector of the target at time-step $k$ is defined as a vector of dimension $2 N$, where $N-1$ is the highest-order time derivative of the target position described by the known target stochastic motion model, and can include components such as position, velocity, acceleration, etc.:

$$
\begin{aligned}
\mathbf{x}_{k} & =\left[\begin{array}{lllllll}
x_{T_{k}} & y_{T_{k}} & \dot{x}_{T_{k}} & \dot{y}_{T_{k}} & \ddot{x}_{T_{k}} & \ddot{y}_{T_{k}} & \cdots
\end{array}\right]^{T} \\
& =\left[\begin{array}{lll}
\mathbf{p}_{T_{k}}^{T} & \mathbf{x}_{T_{\mathbf{d}, k}}^{T}
\end{array}\right]^{T}
\end{aligned}
$$

Here, $\mathbf{p}_{T_{k}} \triangleq\left[\begin{array}{ll}x_{T_{k}} & y_{T_{k}}\end{array}\right]^{T}$ is the target position expressed in the global frame of reference, and $\mathbf{x}_{T_{\mathbf{d}, k}} \triangleq$ $\left[\begin{array}{lllll}\dot{x}_{T_{k}} & \dot{y}_{T_{k}} & \ddot{x}_{T_{k}} & \ddot{y}_{T_{k}} & \cdots\end{array}\right]^{T}$ denotes all the higher-order time derivatives of the target position.

In what follows, we first present the target stochastic motion model and the sensor bearing measurement model that will be used throughout the paper. The standard batch MAP estimator is described afterwards.

\section{A. Motion Model}

We consider the case where the target moves randomly but assume that the stochastic model describing the motion of the target (e.g., constant acceleration or constant velocity [2]) is known. In particular, the discrete-time state propagation equation is generically given by the following linear form:

$$
\mathbf{x}_{k}=\boldsymbol{\Phi}_{k-1} \mathbf{x}_{k-1}+\mathbf{G}_{k-1} \mathbf{w}_{k-1}
$$

where $\mathbf{w}_{k-1}$ is zero-mean white Gaussian noise with covariance $\mathbf{Q}_{k-1}$. The state transition matrix, $\mathbf{\Phi}_{k-1}$, and the process noise Jacobian, $\mathbf{G}_{k-1}$, that appear in the preceding expressions depend on the motion model used [2]. We will make no further assumptions on these matrices other than that their values are known.

\section{B. Measurement Model}

In this work, we focus on the nonlinear case where a single sensor measures its bearing angle to the target. The measurement at time-step $k, z_{k}$, is given by:

$$
\begin{aligned}
z_{k} & =\operatorname{atan} 2\left(\left(y_{T_{k}}-y_{S_{k}}\right),\left(x_{T_{k}}-x_{S_{k}}\right)\right)-\phi_{S_{k}}+n_{k} \\
& \triangleq h\left(\mathbf{x}_{k}\right)+n_{k}
\end{aligned}
$$

where $\mathbf{x}_{S_{k}} \triangleq\left[\begin{array}{ll}\mathbf{p}_{S_{k}}^{T} & \phi_{S_{k}}\end{array}\right]^{T \triangleq} \triangleq\left[\begin{array}{lll}x_{S_{k}} & y_{S_{k}} & \phi_{S_{k}}\end{array}\right]^{T}$ is the known sensor pose expressed in the global frame of reference, and $n_{k}$ is zero-mean white Gaussian measurement noise, with variance $\sigma_{\theta_{k}}^{2}$, i.e., $n_{k} \sim \mathcal{N}\left(n_{k} ; 0, \sigma_{\theta_{k}}^{2}\right)$. 


\section{Batch MAP Estimator}

The batch MAP estimator utilizes all available information to estimate the entire history of the target states, given by stacking all states (1) in the time interval $[0, k]$ :

$$
\mathbf{x}_{0: k}=\left[\begin{array}{llll}
\mathbf{x}_{0}^{T} & \mathbf{x}_{1}^{T} & \cdots & \mathbf{x}_{k}^{T}
\end{array}\right]^{T}
$$

Specifically, this estimator seeks to determine the entire statespace trajectory estimate $\hat{\mathbf{x}}_{0: k \mid k}$ that maximizes the following posterior pdf: ${ }^{1}$

$$
p\left(\mathbf{x}_{0: k} \mid z_{0: k}\right) \propto p\left(\mathbf{x}_{0}\right) \prod_{\kappa=1}^{k} p\left(z_{\kappa} \mid \mathbf{x}_{\kappa}\right) p\left(\mathbf{x}_{\kappa} \mid \mathbf{x}_{\kappa-1}\right)
$$

where $p\left(\mathbf{x}_{0}\right)=\mathcal{N}\left(\mathbf{x}_{0} ; \hat{\mathbf{x}}_{0 \mid 0}, \mathbf{P}_{0 \mid 0}\right)$ is the prior distribution, $z_{0: k}$ denotes all the sensor measurements in the time interval $[0, k]$. For Gaussian state and measurement noise (see (3) and (5), respectively), maximization of (7) is equivalent to minimizing the following cost function [16]:

$$
\begin{aligned}
c\left(\mathbf{x}_{0: k}\right) & =\frac{1}{2}\left\|\mathbf{x}_{0}-\hat{\mathbf{x}}_{0 \mid 0}\right\|_{\mathbf{P}_{0 \mid 0}}^{2}+\sum_{\kappa=1}^{k} \frac{1}{2}\left\|\mathbf{x}_{\kappa}-\mathbf{\Phi}_{\kappa-1} \mathbf{x}_{\kappa-1}\right\|_{\mathbf{Q}_{\kappa-1}^{\prime}}^{2} \\
& +\sum_{\kappa=1}^{k} \frac{1}{2}\left\|z_{\kappa}-h\left(\mathbf{x}_{\kappa}\right)\right\|_{\sigma_{\theta_{\kappa}}^{2}}^{2}
\end{aligned}
$$

where $\|\mathbf{a}\|_{\mathbf{M}}^{2}=\mathbf{a}^{T} \mathbf{M}^{-1} \mathbf{a}$ and $\mathbf{Q}_{k}^{\prime}=\mathbf{G}_{k} \mathbf{Q}_{k} \mathbf{G}_{k}^{T}$ (see (3)).

Since $c\left(\mathbf{x}_{0: k}\right)$ is a nonlinear function, a standard approach for its optimization is to employ Newton-Raphson iterative minimization [17]. At the $\ell$-th iteration of this method, a correction, $\delta \mathbf{x}_{0: k}^{(\ell)}$, to the current estimate, $\hat{\mathbf{x}}_{0: k \mid k}^{(\ell)}$, is computed by minimizing the second-order Taylor-series approximation of the cost function given by:

$c\left(\hat{\mathbf{x}}_{0: k \mid k}^{(\ell)}+\delta \mathbf{x}_{0: k}^{(\ell)}\right) \simeq c\left(\hat{\mathbf{x}}_{0: k \mid k}^{(\ell)}\right)+\mathbf{b}^{(\ell)^{T}} \delta \mathbf{x}_{0: k}^{(\ell)}+\frac{1}{2} \delta \mathbf{x}_{0: k}^{(\ell) T} \mathbf{A}^{(\ell)} \delta \mathbf{x}_{0: k}^{(\ell)}$

where $\mathbf{b}^{(\ell)}$ and $\mathbf{A}^{(\ell)}$ are the Jacobian and Hessian of $c(\cdot)$ with respect to $\mathbf{x}_{0: k}$, evaluated at $\hat{\mathbf{x}}_{0: k \mid k}^{(\ell)}$, respectively (see [16]). The value $\delta \mathbf{x}_{0: k}^{(\ell)}$ that minimizes (9) is found by solving the linear system:

$$
\mathbf{A}^{(\ell)} \delta \mathbf{x}_{0: k}^{(\ell)}=-\mathbf{b}^{(\ell)}
$$

Once $\delta \mathbf{x}_{0: k}^{(\ell)}$ is found, the new state estimate is computed as:

$$
\hat{\mathbf{x}}_{0: k \mid k}^{(\ell+1)}=\hat{\mathbf{x}}_{0: k \mid k}^{(\ell)}+\delta \mathbf{x}_{0: k}^{(\ell)}
$$

Given an initial estimate $\hat{\mathbf{x}}_{0: k \mid k}^{(0)}$, this iterative algorithm converges to a stationary point, but cannot provide guarantees about global optimality.

\section{InCREMENTALly SOLVING THE BATCH MAP OPTIMIZATION PROBLEM}

In this section, we present an incremental solution to the batch MAP problem of minimizing (8). Since it is, in general, intractable to analytically solve the batch MAP problem, we relax it by holding previous state estimates constant and incrementally solving a one-step minimization problem for

\footnotetext{
${ }^{1}$ Throughout the paper, the subscript $\ell \mid j$ refers to the estimate of a quantity at time-step $\ell$, after all measurements up to time-step $j$ have been processed. $\hat{x}$ is used to denote the estimate of a random variable $x$, while $\tilde{x}=x-\hat{x}$ is the error in this estimate. $\mathbf{I}_{n}$ is the $n \times n$ identity matrix.
}

the current state estimate analytically, at every time step when a new measurement becomes available. This analytic optimization is efficiently carried out by transforming the bearing measurement and thus the nonlinear cost function into rational (polynomial) forms.

\section{A. Relaxation of the Batch MAP Problem}

By transforming the trigonometric function of the bearing measurement (4) into rational form, we can convert the KKT optimality conditions of the batch MAP problem into a polynomial system that has $2 N(k+1)$ variables (see Section IV-B). It is clear that the number of variables increases linearly with respect to the time horizon $k$. However, since the complexity of solving multivariate polynomial systems is exponential in the number of variables [18], in general, it is computationally intractable to solve the batch MAP problem analytically.

For this reason, we relax the batch MAP problem and solve it incrementally. We start by noting that the cost function (8) can be written as:

$$
\begin{aligned}
c\left(\mathbf{x}_{0: k}\right) & =c\left(\mathbf{x}_{0: k-1}\right)+\frac{1}{2}\left\|\mathbf{x}_{k}-\mathbf{\Phi}_{k-1} \mathbf{x}_{k-1}\right\|_{\mathbf{Q}_{k-1}^{\prime}}^{2} \\
& +\frac{1}{2}\left\|z_{k}-h\left(\mathbf{x}_{k}\right)\right\|_{\sigma_{\theta_{k}}^{2}}^{2}
\end{aligned}
$$

Since the motion model (3) is linear, at every time step when a new measurement becomes available, we can combine all previous information about the target's trajectory to provide a prior for the new current state, $\mathbf{x}_{k}$, based on the following propagation equations:

$$
\begin{aligned}
\hat{\mathbf{x}}_{k \mid k-1} & =\boldsymbol{\Phi}_{k-1} \hat{\mathbf{x}}_{k-1 \mid k-1} \\
\mathbf{P}_{k \mid k-1} & =\boldsymbol{\Phi}_{k-1} \mathbf{P}_{k-1 \mid k-1} \boldsymbol{\Phi}_{k-1}^{T}+\mathbf{G}_{k-1} \mathbf{Q}_{k-1} \mathbf{G}_{k-1}^{T}
\end{aligned}
$$

Thus, the new state estimate can be computed incrementally by solving the following one-step minimization problem:

$$
\min _{\mathbf{x}_{k}}\left[\frac{1}{2}\left\|\mathbf{x}_{k}-\hat{\mathbf{x}}_{k \mid k-1}\right\|_{\mathbf{P}_{k \mid k-1}}^{2}+\frac{1}{2}\left\|z_{k}-h\left(\mathbf{x}_{k}\right)\right\|_{\sigma_{\theta_{k}}^{2}}^{2}\right]
$$

\section{B. Analytic Determination of the Local Minima}

Observing that the bearing measurement depends only on the target position (see (4)), we can decouple the target position, $\mathbf{p}_{T_{k}}$, and the remaining states, $\mathbf{x}_{T_{\mathrm{d}, k}}$, in solving (15), so as to simplify the ensuing derivations. Specifically, using the following partitioning of the information matrix of the full state, $\mathbf{P}_{k \mid k-1}^{-1}=\left[\begin{array}{ll}\boldsymbol{\Sigma}_{\mathbf{p p}_{k \mid k-1}} & \boldsymbol{\Sigma}_{\mathbf{p d}_{k \mid k-1}} \\ \boldsymbol{\Sigma}_{\mathbf{d p}_{k \mid k-1}} & \boldsymbol{\Sigma}_{\mathbf{d d}_{k \mid k-1}}\end{array}\right]$, the cost function of (15) can be written as:

$$
\begin{aligned}
c\left(\mathbf{x}_{k}\right)= & \frac{1}{2}\left(\mathbf{p}_{T_{k}}-\hat{\mathbf{p}}_{T_{k \mid k-1}}\right)^{T} \boldsymbol{\Sigma}_{\mathbf{p p}_{k \mid k-1}}\left(\mathbf{p}_{T_{k}}-\hat{\mathbf{p}}_{T_{k \mid k-1}}\right)+ \\
& \frac{1}{2}\left(\mathbf{x}_{T_{\mathbf{d}, k}}-\hat{\mathbf{x}}_{T_{\mathbf{d}, k \mid k-1}}\right)^{T} \boldsymbol{\Sigma}_{\mathbf{d d _ { k } | k - 1}}\left(\mathbf{x}_{T_{\mathbf{d}, k}}-\hat{\mathbf{x}}_{T_{\mathbf{d}, k \mid k-1}}\right)+ \\
& \left(\mathbf{p}_{T_{k}}-\hat{\mathbf{p}}_{T_{k \mid k-1}}\right)^{T} \boldsymbol{\Sigma}_{\mathbf{p d}} \mathbf{d}_{k \mid k-1}\left(\mathbf{x}_{T_{\mathbf{d}, k}}-\hat{\mathbf{x}}_{T_{\mathbf{d}, k \mid k-1}}\right)+ \\
& \frac{1}{2 \sigma_{\theta_{k}}^{2}}\left(z_{k}-h\left(\mathbf{p}_{T_{k}}\right)\right)^{2}
\end{aligned}
$$

We note that

$$
\min _{\mathbf{p}_{T_{k}}, \mathbf{x}_{T_{\mathbf{d}}, k}} c\left(\mathbf{p}_{T_{k}}, \mathbf{x}_{T_{\mathbf{d}, k}}\right)=\min _{\mathbf{p}_{T_{k}}}\left(\min _{\mathbf{x}_{T_{\mathbf{d}}, k}} c\left(\mathbf{p}_{T_{k}}, \mathbf{x}_{T_{\mathbf{d}, k}}\right)\right)
$$


Thus, we first solve for $\mathbf{x}_{T_{\mathbf{d}, k}}$ by setting the gradient of (16) with respect to $\mathbf{x}_{T_{\mathbf{d}, k}}$ to zero, and obtain:

$$
\mathbf{x}_{T_{\mathbf{d}, k}}=\hat{\mathbf{x}}_{T_{\mathbf{d}, k \mid k-1}}-\boldsymbol{\Sigma}_{\mathbf{d d}_{k \mid k-1}}^{-1} \boldsymbol{\Sigma}_{\mathbf{d p}_{k \mid k-1}}\left(\mathbf{p}_{T_{k}}-\hat{\mathbf{p}}_{T_{k \mid k-1}}\right)
$$

Substitution of (17) into (16) yields:

$$
\begin{aligned}
c\left(\mathbf{p}_{T_{k}}\right)= & \frac{1}{2}\left(\mathbf{p}_{T_{k}}-\hat{\mathbf{p}}_{T_{k \mid k-1}}\right)^{T} \mathbf{P}_{\mathbf{p} \mathbf{p}_{k \mid k-1}}^{-1}\left(\mathbf{p}_{T_{k}}-\hat{\mathbf{p}}_{T_{k \mid k-1}}\right)+ \\
& \frac{1}{2 \sigma_{\theta_{k}}^{2}}\left(z_{k}-h\left(\mathbf{p}_{T_{k}}\right)\right)^{2}
\end{aligned}
$$

where $\mathbf{P}_{\mathbf{p p}_{k \mid k-1}}$ is the covariance matrix corresponding to the target position, obtained by partitioning the covariance matrix as $\mathbf{P}_{k \mid k-1}=\left[\begin{array}{ll}\mathbf{P}_{\mathbf{p p}_{k \mid k-1}} & \mathbf{P}_{\mathbf{p d}_{k \mid k-1}} \\ \mathbf{P}_{\mathbf{d p}_{k \mid k-1}} & \mathbf{P}_{\mathbf{d d}_{k \mid k-1}}\end{array}\right]$. In (18), we have employed the identity, $\mathbf{P}_{\mathbf{p} \mathbf{p}_{k \mid k-1}}^{-1}=\boldsymbol{\Sigma}_{\mathbf{p p}_{k \mid k-1}}$ $\boldsymbol{\Sigma}_{\mathbf{p d}_{k \mid k-1}} \boldsymbol{\Sigma}_{\mathbf{d d}_{k \mid k-1}}^{-1} \boldsymbol{\Sigma}_{\mathbf{d p}_{k \mid k-1}}$, which can easily be verified using the block matrix inversion lemma [19].

We thus see that the problem (15) can be decoupled as follows: We first solve for $\hat{\mathbf{p}}_{T_{k}}$ by minimizing (18), and from that we then solve linearly for $\hat{\mathbf{x}}_{T_{\mathrm{d}, k}}$ using (17). It is also important to note that the size of the nonlinear problem has dramatically decreased, from $2 N$, for (15), to a constant size of 2 for minimizing (18). Moreover, the analytic solution for the target position is independent of its higher-order time derivatives, regardless of the stochastic target motion model.

Now we focus on the analytic solution of (18). In order to use an algebraic geometry approach, we transform the bearing measurement function (4) into a rational form. Specifically, after moving the sensor orientation term to the right hand side of (4), we apply the tangent function on both sides and obtain the following transformed measurement:

$$
\begin{aligned}
\check{z}_{k} & \triangleq \tan \left(z_{k}+\phi_{S_{k}}\right) \\
& =\tan \left(\operatorname{atan} 2\left(\left(y_{T_{k}}-y_{S_{k}}\right),\left(x_{T_{k}}-x_{S_{k}}\right)\right)+n_{k}\right)
\end{aligned}
$$

By denoting $\xi_{k} \triangleq \operatorname{atan} 2\left(\left(y_{T_{k}}-y_{S_{k}}\right),\left(x_{T_{k}}-x_{S_{k}}\right)\right)$, considering $z_{k}+\phi_{S_{k}} \in(-\pi, \pi]$, and following the standard formulae to compute the pdf of functions of random variables [20], the pdf of the transformed measurement can be computed as:

$$
\begin{aligned}
& p\left(\check{z}_{k} \mid \mathbf{x}_{k}\right)= \\
& \begin{cases}\frac{\mathcal{N}\left(\tan ^{-1}\left(\check{z}_{k}\right) ; \xi_{k}, \sigma_{\theta_{k}}^{2}\right)+\mathcal{N}\left(\tan ^{-1}\left(\check{z}_{k}\right)-\pi ; \xi_{k}, \sigma_{\theta_{k}}^{2}\right)}{1+\check{z}_{k}^{2}}, & \text { if } \check{z}_{k} \geq 0 \\
\frac{\mathcal{N}\left(\tan ^{-1}\left(\check{z}_{k}\right) ; \xi_{k}, \sigma_{\theta_{k}}^{2}\right)+\mathcal{N}\left(\tan ^{-1}\left(\check{z}_{k}\right)+\pi ; \xi_{k}, \sigma_{\theta_{k}}^{2}\right)}{1+\check{z}_{k}^{2}}, & \text { if } \check{z}_{k}<0\end{cases}
\end{aligned}
$$

Clearly, $p\left(\check{z}_{k} \mid \mathbf{x}_{k}\right)$ is not Gaussian (which results from the tangent of a Gaussian random variable), but it can be well approximated by a Gaussian pdf by matching first and second order moments. This is done by linearizing (19) around the expected value of the noise, i.e.,

$$
\check{z}_{k} \simeq \frac{y_{T_{k}}-y_{S_{k}}}{x_{T_{k}}-x_{S_{k}}}+\bar{n}_{k} \triangleq \bar{z}_{k}
$$

where $\bar{n}_{k} \triangleq \sec ^{2}\left(z_{k}+\phi_{S_{k}}\right) n_{k}$, is zero-mean white Gaussian noise with variance $\sigma_{k}^{2} \triangleq \sec ^{4}\left(z_{k}+\phi_{S_{k}}\right) \sigma_{\theta_{k}}^{2}$, i.e., $\bar{n}_{k} \sim$ $\mathcal{N}\left(0, \sigma_{k}^{2}\right)$. We term this approximation (21) an inferred measurement which is in the desired rational form. As illustrated in Fig. 1, this approximation is reasonably accurate, particularly for high signal-to-noise ratios. Moreover, the local minimum of (18) attained based on the inferred measurement

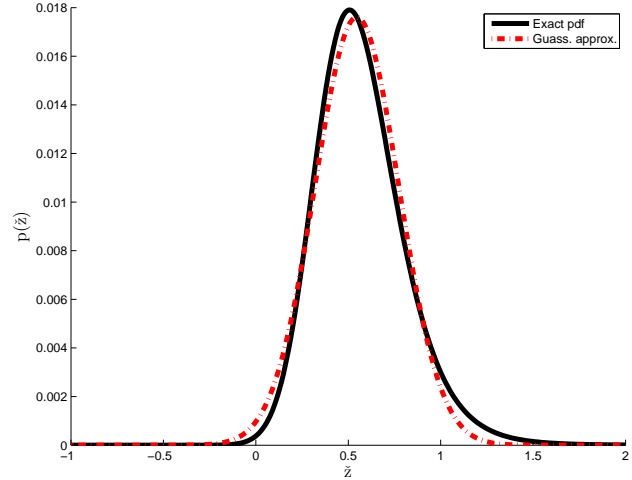

Fig. 1. An example of approximating the pdf of transformed measurements by a Gaussian pdf. In this case, $\xi=0.5$ and $\sigma_{\theta}=10 \mathrm{deg}$. In addition, the Kullback-Leibler divergence (KLD) between these two pdfs is only 0.0447 , which indicates the difference between the two distributions is small.

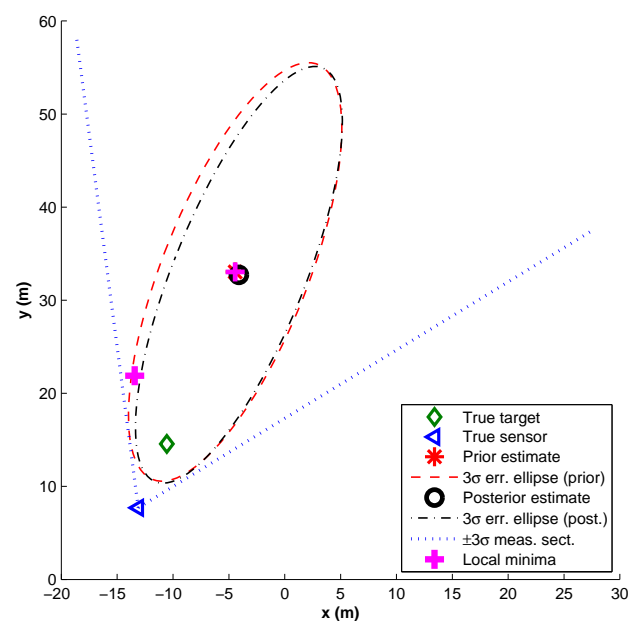

Fig. 2. Illustrative problem for a single time step bearing-only tracking: The magenta crosses indicate the locations of the analytically computed local minima (25)-(26). The MAP estimate initialized with the prior estimate converges to the local minimum with larger error with respect to ground truth. Note that the MAP estimate is computed based on the original (not inferred) measurements. The approximation (21) used in the inferred measurements introduces a slight offset in the analytic local minima.

is very close to that based on the corresponding original bearing measurement. This can be seen from Fig. 2, where one of the local minima using the inferred measurement almost coincides with the IEKF posterior estimate (which corresponds to the local minimum determined by the GaussNewton method [21]) using the original bearing measurement. This further shows that the inferred measurement is a reasonably good approximation to the original measurement. ${ }^{2}$ Moreover, the inferred measurement is used only for finding the hypotheses of the trajectory, not for estimating the states, whose estimates will be updated by the batch MAP estimators using all available original bearing measurements.

In what follows we use the inferred measurement (21) (instead of (5)) to compute the analytic solutions for minimizing (18). In particular, with $\mathbf{P}_{\mathbf{p} \mathbf{p}_{k \mid k-1}}^{-1}=\left[\begin{array}{ll}s_{1} & s_{3} \\ s_{3} & s_{2}\end{array}\right]$,

\footnotetext{
${ }^{2}$ Also note that this inferred measurement model (21) does not consider the special case of $x_{T_{k}}=x_{S_{k}}$. However, this case has low probability of occurrence in practice, and can be easily avoided through an appropriate coordinate transformation.
} 
can be written as:

$$
\begin{aligned}
& c\left(x_{T_{k}}, y_{T_{k}}\right)=\frac{1}{2}\left(s_{1}\left(x_{T_{k}}-\hat{x}_{T_{k \mid k-1}}\right)^{2}+s_{2}\left(y_{T_{k}}-\hat{y}_{T_{k \mid k-1}}\right)^{2}+\right. \\
& \left.2 s_{3}\left(x_{T_{k}}-\hat{x}_{T_{k \mid k-1}}\right)\left(y_{T_{k}}-\hat{y}_{T_{k \mid k-1}}\right)\right)+\frac{1}{2 \sigma_{k}^{2}}\left(\bar{z}_{k}-\frac{y_{T_{k}}-y_{S_{k}}}{x_{T_{k}}-x_{S_{k}}}\right)^{2}
\end{aligned}
$$

Setting the derivatives of $c\left(x_{T_{k}}, y_{T_{k}}\right)$ with respect to the two optimization variables to zero, and performing simple algebraic manipulations, we have:

$$
\begin{aligned}
& \frac{\partial c}{\partial x_{T_{k}}}=0 \Rightarrow \\
& s_{1}\left(x_{T_{k}}-\hat{x}_{T_{k \mid k-1}}\right)\left(x_{T_{k}}-x_{S_{k}}\right)^{3}+ \\
& s_{3}\left(x_{T_{k}}-x_{S_{k}}\right)^{3}\left(y_{T_{k}}-\hat{y}_{T_{k \mid k-1}}\right)+ \\
& \frac{1}{\sigma_{k}^{2}}\left[\bar{z}_{k}\left(x_{T_{k}}-x_{S_{k}}\right)\left(y_{T_{k}}-y_{S_{k}}\right)-\left(y_{T_{k}}-y_{S_{k}}\right)^{2}\right]=0 \\
& \frac{\partial c}{\partial y_{T_{k}}}=0 \Rightarrow \\
& s_{2}\left(x_{T_{k}}-x_{S_{k}}\right)^{2}\left(y_{T_{k}}-\hat{y}_{T_{k \mid k-1}}\right)+ \\
& s_{3}\left(x_{T_{k}}-x_{S_{k}}\right)^{2}\left(x_{T_{k}}-\hat{x}_{T_{k \mid k-1}}\right)- \\
& \frac{1}{\sigma_{k}^{2}}\left[\bar{z}_{k}\left(x_{T_{k}}-x_{S_{k}}\right)-\left(y_{T_{k}}-y_{S_{k}}\right)\right]=0
\end{aligned}
$$

From (24), we can compute $y_{T_{k}}$ in terms of $x_{T_{k}}$ as follows:

$$
\begin{aligned}
y_{T_{k}}= & \frac{-\sigma_{k}^{2} s_{3}\left(x_{T_{k}}-x_{S_{k}}\right)^{2}\left(x_{T_{k}}-\hat{x}_{T_{k \mid k-1}}\right)+\bar{z}_{k}\left(x_{T_{k}}-x_{S_{k}}\right)}{1+\sigma_{k}^{2} s_{2}\left(x_{T_{k}}-x_{S_{k}}\right)^{2}}+ \\
& \frac{\sigma_{k}^{2} s_{2}\left(x_{T_{k}}-x_{S_{k}}\right)^{2} \hat{y}_{T_{k \mid k-1}}+y_{S_{k}}}{1+\sigma_{k}^{2} s_{2}\left(x_{T_{k}}-x_{S_{k}}\right)^{2}}
\end{aligned}
$$

Substitution of (25) into (23) yields a rational equation, whose denominator is always non-zero. Thus, we only need to focus on the numerator, which is an eighth-order univariate polynomial in $x_{T_{k}}$ :

$$
0=f\left(x_{T_{k}}\right)=\sum_{i=0}^{8} a_{i} x_{T_{k}}^{i}
$$

where the coefficients $a_{i}, i=0, \ldots, 8$, are expressed in terms of the known quantities, $\bar{z}_{k}, \sigma_{k}, s_{1}, s_{2}, s_{3}, \hat{x}_{T_{k \mid k-1}}$, $\hat{y}_{T_{k \mid k-1}}, x_{S_{k}}$, and $y_{S_{k}}$ [16]. The roots of $f\left(x_{T_{k}}\right)$ can be found from the eigenvalues of the corresponding $8 \times 8$ companion matrix [22].

Although there exist 8 solutions for $x_{T_{k}}$ and thus 8 solutions for $y_{T_{k}}$, as it depends injectively on $x_{T_{k}}$ (see (25)), we only need to consider the pairs $\left(x_{T_{k}}, y_{T_{k}}\right)$ that correspond to real eigenvalues of the companion matrix. Moreover, since some of these solutions could be local maxima or saddle points, the second-order derivative test [23] is employed to extract the minima. Finally, once we determine all the local minima for the target position, we compute the corresponding estimates for the higher-order position derivatives via (17).

The following lemma provides a tighter upper bound for the maximum number of local minima, which is important since it significantly impacts the computational complexity of our proposed method (see Section V).

Lemma 4.1: There exist at most 7 local minima for (22).

Proof: Using the Finite Dimensional Mountain Pass Theorem (see Theorem 5.2 in [24]), which states that there exists a third critical point which is not a local minimum between any two strict local minima for a coercive $\mathcal{C}^{1}$ function, ${ }^{3}$ it is straightforward to show that our cost function (22) $\in \mathcal{C}^{1}\left(\mathbb{R}^{2} \backslash\left\{x_{T_{k}}=x_{S_{k}}\right\}\right)$ is coercive, and thus at least one of the 8 critical points cannot be a local minimum, leaving a maximum number of 7 local minima.

Note that due to its rational form, the inferred measurement (21) is symmetric with respect to the sensor, which, however, is not the case for the original bearing measurement (5). This can result in more local minima of (22) than those of (18). To discard the spurious local minima due to the symmetry of the inferred measurement, we employ the Mahalanobis distance test [2]. As a result, we have never observed more than four local minima in practice.

\section{A BANK OF MAP ESTIMATORS}

As discussed in the preceding section, due to the nonlinearity of bearing measurements, the incremental one-step MAP problem (15) and thus the original multi-step MAP problem (8), has multiple local minima that correspond to the modes of the posterior pdf. Any iterative algorithm (e.g., Gauss-Newton) used in the batch MAP estimator only converges to the global optimum and hence the true MAP estimate, if the initial estimate $\hat{\mathbf{x}}_{0: k \mid k}^{(0)}$ is within the region of attraction of the global optimum. However, in general, there exists no systematic method for determining such an initial estimate that can ensure convergence to the global optimum. As a result, the standard batch MAP estimator when used for target tracking can become inconsistent and even diverge if no good initial estimate is provided. This is confirmed by the simulation and experimental results presented in Sections VI and VII.

To mitigate this issue, in this section, we propose a general estimation framework for tracking multiple local minima, and specialize it to the case of bearing-only tracking. The key idea of this approach is to use the analytically computed local minima at each time step (see Section IV-B) as guidance to find and track most probable hypotheses of the target trajectory, thus improving tracking performance.

Specifically, at time-step $k-1$, based on (13) and (14), we first propagate the current state estimate, $\hat{\mathbf{x}}_{k-1 \mid k-1}^{[i]}$ corresponding to the $i$-th solution and its covariance matrix $\mathbf{P}_{k-1 \mid k-1}^{[i]}, \forall i=1,2, \ldots, m$ ( $m$ is number of estimators in the bank at time-step $k-1$ ). Then, once a new measurement becomes available, the propagated state estimate and covariance, $\hat{\mathbf{x}}_{k \mid k-1}^{[i]}$ and $\mathbf{P}_{k \mid k-1}^{[i]}$, are used as the prior in (15). Next, we use the algebraic geometry method presented in Section IV-B to analytically determine all the local minima of (15), denoted by $\mathbf{x}_{k}^{[j]}, 1 \leq j \leq 7 m$ (see Lemma 4.1). Finally, for each of these solutions, we employ a batch MAP estimator that uses the latest estimates of the trajectory corresponding to this solution as the initial value, to refine the entire state estimates $\hat{\mathbf{x}}_{0: k \mid k}^{[j]}$ up to current time-step $k$ (see (8)). This procedure recursively evolves over time, and at every time step, generates at most $7 m$ trajectory estimates. In

\footnotetext{
${ }^{3}$ A function $f: \mathbb{R}^{N} \rightarrow \mathbb{R}$ is coercive if it is bounded from below and is proper in the sense that $f(\mathbf{x}) \rightarrow \infty$ for $\|\mathbf{x}\| \rightarrow \infty$.
} 
the end, we will have multiple MAP estimates, among which the one with the least cost is selected as the best estimate for the global optimum (and thus for the true state). Algorithm 1 outlines the main steps of the proposed algorithm.

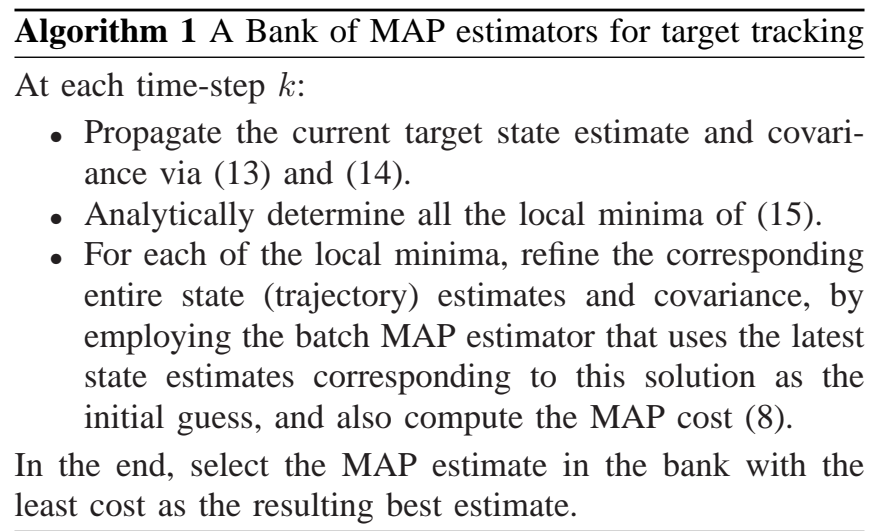

We should point out that, in the worst case, the total number of solutions, and thus MAP estimators in the bank, grows exponentially with time. However, in practice the number of physically different trajectory hypotheses is significantly lower, since many different initial guesses reside within the same basin of attraction. In addition, as the target continuously moves, the size of the state vector $\mathbf{x}_{0: k}^{[i]}$ of each MAP estimator increases linearly with time. So, in order to reduce the computational cost of the proposed algorithm, as in our previous work [9], we employ a pruning scheme by keeping at most $m_{\max }$ estimators in the bank, as well as marginalization of old states by maintaining a sliding window of states. The details of these two processes can be found in [9], [16].

\section{Simulation Results}

A series of Monte Carlo simulations were conducted under various conditions, in order to demonstrate the capability of the proposed bank of MAP estimators to improve tracking performance. The metrics used to evaluate estimator's performance were: (i) the average root mean squared error (RMSE), and (ii) the normalized (state) estimation error squared (NEES) [2]. Both metrics are computed by averaging over all Monte Carlo runs for each time step. The average RMSE provides us with a concise metric of the accuracy of a given estimator, while the NEES is a metric for evaluating the estimator's consistency. By studying both the RMSE and NEES of all the estimators under consideration, we obtain a comprehensive picture of the estimators' performance.

In the following simulation tests, we performed 100 Monte Carlo simulations, and compared several different estimators. During each Monte Carlo run, all the estimators process the same data, to ensure a fair comparison. The compared estimators were: (i) the standard EKF, (ii) the standard batch MAP estimator using the EKF estimates as the initial value as well as employing the marginalization process as in (iv), (iii) the sampling importance resampling (SIR)-PF with 3000 particles [7], and (iv) the proposed bank of MAP estimators with pruning $\left(m_{\max }=10\right)$ and marginalization (sliding window of 20 time steps). Note that in both MAP estimators, the maximum number of Gauss-Newton iterations allowed is set to 50. Note also that depending on different resampling schemes and different proposal distributions used by the $\mathrm{PF}$, many variants exist, e.g., auxiliary PF, regularized PF, likelihood PF, etc. Interested readers are referred to [7], [15], [25] for an overview of the PF. In this simulation, we implemented the standard (conventional) SIR-PF that uses the state-transition prior distribution as the proposal distribution and employs systematic resampling at every time step. Moreover, to alleviate the particle depletion problem, we also dithered the process noise (increase noise covariance). We also examined different resampling schemes such as Ripley's and stratified resampling [15], but found negligible performance improvement.

For the results presented in this section, we adopted a zero-acceleration motion model for the target [2]:

$$
\dot{\mathbf{x}}(t)=\mathbf{F} \mathbf{x}(t)+\mathbf{G w}(t)
$$

where

$$
\mathbf{F}=\left[\begin{array}{cccc}
0 & 0 & 1 & 0 \\
0 & 0 & 0 & 1 \\
0 & 0 & 0 & 0 \\
0 & 0 & 0 & 0
\end{array}\right], \mathbf{G}=\left[\begin{array}{cc}
0 & 0 \\
0 & 0 \\
1 & 0 \\
0 & 1
\end{array}\right], \mathbf{x}(t)=\left[\begin{array}{c}
x_{T}(t) \\
y_{T}(t) \\
\dot{x}_{T}(t) \\
\dot{y}_{T}(t)
\end{array}\right]
$$

and $\mathbf{w}(t)=\left[\begin{array}{ll}w_{x}(t) & w_{y}(t)\end{array}\right]^{T}$ is zero-mean, white Gaussian noise with covariance $\mathbb{E}\left[\mathbf{w}(t) \mathbf{w}(\tau)^{T}\right]=q \mathbf{I}_{2} \delta(t-\tau)$, where $q=2\left(\frac{\mathrm{m}}{\mathrm{sec}}\right)^{2} \frac{1}{\mathrm{~Hz}}$, and $\delta(t-\tau)$ is the Dirac delta function. In the implementation, we discretized this continuous-time system model (27) with time step $\Delta t=0.1 \mathrm{sec}$. The initial true target state was $\mathbf{x}_{0}=\left[\begin{array}{llll}0 & 0 & -5 & 5\end{array}\right]^{T}$, while the initial estimate of the target state was randomly generated from a Gaussian pdf, $\mathcal{N}\left(\hat{\mathbf{x}}_{0 \mid 0} ; \mathbf{x}_{0}, \mathbf{P}_{0 \mid 0}\right)$, where $\mathbf{P}_{0 \mid 0}=10^{3} \mathbf{I}_{4}$ is the initial covariance of the state estimate. Similar to [10], we chose a circular sensor trajectory with perfectly known poses for this simulation. Fig. 3(a) shows the trajectories of the target and sensor in one typical realization of Monte Carlo simulations. In this simulation, the standard deviation of the bearing-measurement noise was set to $10 \mathrm{deg}$.

Fig. 3 shows the Monte Carlo results of the four estimators. As evident from this figure, the standard EKF estimates are inaccurate (RMSE), diverge from the ground truth, and become inconsistent (NEES). In contrast, the continuous relinearization of the standard batch MAP estimator that incrementally uses the EKF estimates as the initial guess, significantly improves its performance. As expected, the PF attains better estimation accuracy than the EKF. This is due to the fact that each particle in the PF essentially represents a hypothesis of the target state, and thus the PF is more likely to converge to the optimal solution. However, it does not work as well as the standard batch MAP estimator, in part because it does not allow smoothing of the old state estimates using new available measurements. ${ }^{4}$ Note also that the NEES of the PF is not necessarily better than that of the EKF, primarily due to the fact that the covariance matrices

\footnotetext{
${ }^{4}$ Although particle-based smoothers exist, their computational requirements are significantly higher [15].
} 


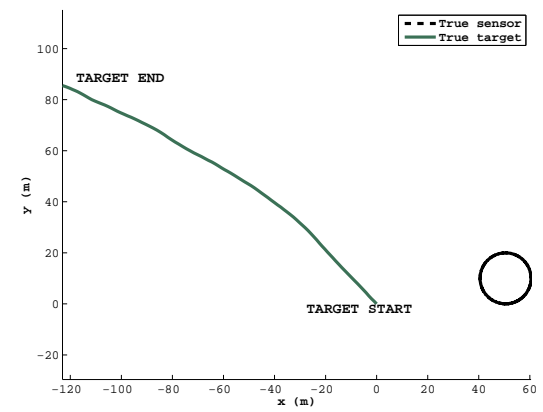

(a)
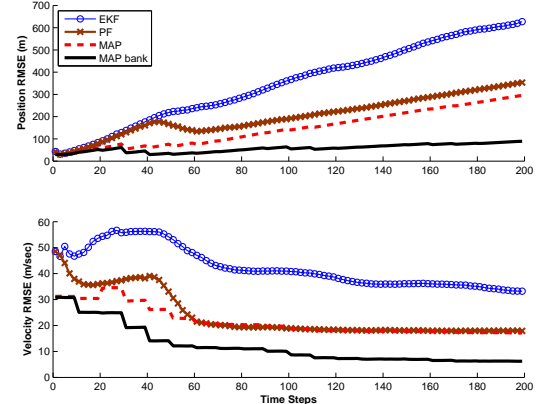

(b)

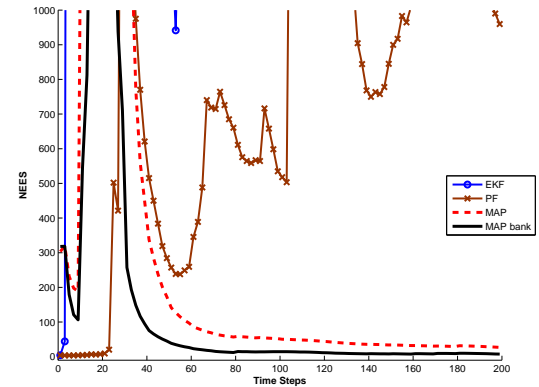

(c)

Fig. 3. Monte Carlo simulation results: (a) The trajectories of the target and sensor obtained from one typical realization of the 100 Monte Carlo simulations, (b) RMSE, (c) NEES. It is clear that the proposed algorithm performs substantially better than its competitors, in terms of both accuracy (RMSE) and consistency (NEES). Note that for clarity of presentation, only portions of the NEES lines that are within certain thresholds are plotted.

of the PF computed from particles in some trials become illconditioned. Most importantly, the bank of MAP estimators performs substantially better than its competitors, in terms of both accuracy and consistency. This is attributed to the good initial estimates attained by analytically computing the modes of the posterior pdf (see Section IV-B).

Finally, using the same simulation setup as described above, we compared the computational requirements of the proposed bank of MAP estimators and the PF. In particular, we count the CPU running time for a complete update of the $\mathrm{PF}$ and the bank of MAP estimators (including the analytic determination of all local minima, batch MAP refinement, pruning, and marginalization). Our Matlab implementation running on a Core2 Quad CPU requires an average execution time of $0.57 \mathrm{sec}$ for the bank of MAP, compared to $0.52 \mathrm{sec}$ for the PF. These results are obtained by averaging the CPU running time over all Monte Carlo runs and over all time steps. Note that although the proposed algorithm is slightly more computationally demanding than the PF, it achieves significantly better tracking performance (see Fig. 3(b)). Specifically, the bank of MAP estimators achieves on average $70 \%$ higher position and $50 \%$ higher velocity estimation accuracy compared to the PF, at only $10 \%$ higher computational cost.

\section{EXPERIMENTAL RESULTS}

In this section, we present a real-world experiment performed to further validate the proposed bank of MAP estimators. During the test, two Pioneer-III robots, one acting as the target and the other serving as the sensor, were moving in a rectangular area of $4 \mathrm{~m} \times 2 \mathrm{~m}$, within which the positions of the robots were tracked by an overhead camera. For this purpose, rectangular tracking patterns were mounted on top of the robots and the vision system was calibrated in order to provide ground-truth measurements of the robots' poses in a global coordinate frame. The standard deviation of the noise in these measurements was approximately 0.5 deg for orientation and $0.01 \mathrm{~m}$, along each axis, for position. The sensor robot moved on a circle, while the target robot was commanded to move along a straight line at a constant velocity of $v=0.1 \mathrm{~m} / \mathrm{sec}$. Thus a zero-acceleration motion model with $q=0.01$ was used to describe the target's motion (see (27)). Fig. 4(a) shows the experimental setup,

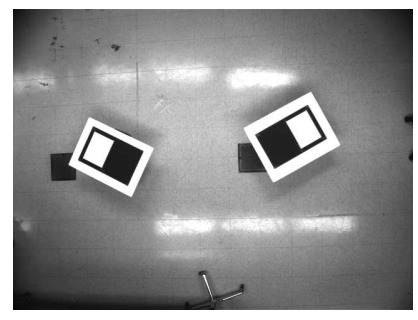

(a)

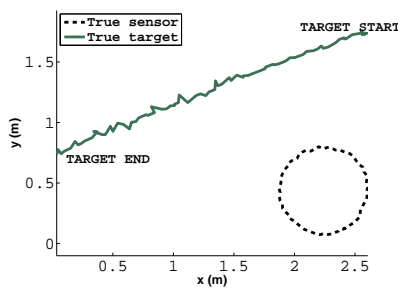

(b)
Fig. 4. Experimental setup: (a) Calibrated image of two Pioneer III robots (one acts as the target while the other is the sensor) with tracking patterns mounted on top of them. (b) Trajectories of the two robots (target and sensor) that move inside a $4 \mathrm{~m} \times 2 \mathrm{~m}$ arena during the indoor experiment.

and Fig. 4(b) depicts the trajectories of the target and sensor.

In this experiment, the initial estimate of the target state was set to $\hat{\mathbf{x}}_{0 \mid 0}=\left[\begin{array}{llll}2.5940 & 1.7374 & 0.0003 & -0.0001\end{array}\right]^{T}$ with covariance $\mathbf{P}_{0 \mid 0}=\mathbf{I}_{4}$. Relative bearing measurements were produced synthetically using the differences in the positions of the target and sensor, as these were recorded by the overhead camera, with the addition of noise. For the experimental results shown in the following, the bearing measurements were corrupted by zero-mean white Gaussian noise, with standard deviation $\sigma_{\theta}=5 \mathrm{deg}$.

The same four estimators as in the simulations were implemented, and the comparative results are presented in Fig. 5. From the experimental results, it becomes clear that the bank of MAP estimators outperforms the standard $\mathrm{EKF}$, the PF, and the batch MAP estimators, in terms of accuracy and consistency. This agrees with the simulation results presented in the preceding section. Thus, both the experimental and simulation results confirm that in nonlinear estimation problems, correctly finding and tracking all modes of the posterior pdf, as well as reducing linearization errors, significantly improves the estimator's performance.

\section{CONCLUSIONS AND FUTURE WORK}

In order to improve consistency, estimators that are applied to nonlinear problems should be able to track multimodal pdfs. However, this is not the case for many existing estimators (e.g., the EKF, and the MAP estimator). In this paper, we have introduced a general estimation framework, a 

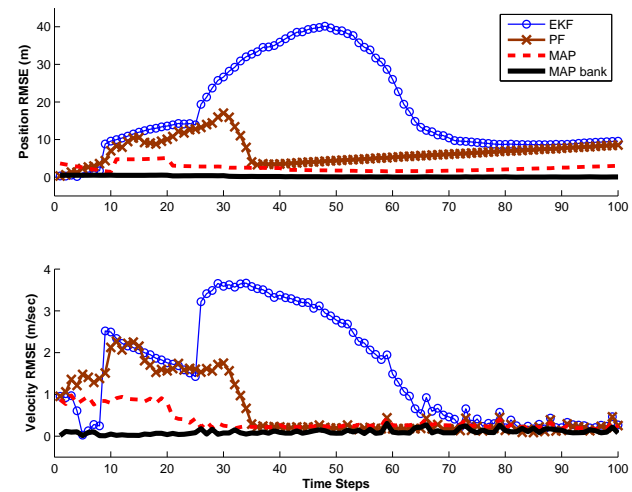

(a)

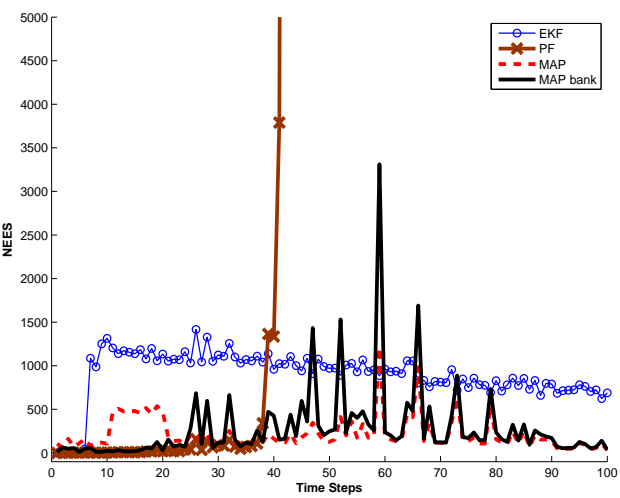

(b)

Fig. 5. Experimental results: (a) RMSE, (b) NEES. Note that for clarity of presentation, only portions of the NEES lines that are within certain thresholds are plotted.

bank of MAP estimators, that simultaneously allows tracking multiple modes of the pdf, and reduces linearization errors through relinearization of past measurements. We have then applied it to the problem of bearing-only tracking. Due to its computational intractability, we have employed a relaxation scheme that keeps past state estimates temporarily constant and incrementally solves a one-step minimization problem for the current state estimate at every time step. This minimization is solved using algebraic geometry methods. The analytically computed local minima are then used to find accurate initial values for the bank of MAP estimators, hence focusing the available resources on tracking the most probable hypotheses of the target trajectory. Additionally, to reduce the computational complexity of the proposed algorithm, we have employed hypothesis pruning along with marginalization of old states. Simulation and experimental results have shown that the proposed algorithm significantly outperforms the standard EKF, the batch MAP estimator, as well as the PF, in terms of both accuracy and consistency.

In future work, we plan to incorporate active sensing (optimal sensor motion control) [26] into the current estimation framework to further improve tracking performance. In addition, the extension of current work to target tracking in $3 \mathrm{D}$ will be of practical importance.

\section{REFERENCES}

[1] S. S. Blackman and R. F. Popoli, Design and Analysis of Modern Tracking Systems. Artech House, 1999.

[2] Y. Bar-Shalom, X. Li, and T. Kirubarajan, Estimation with applications to tracking and navigation. John Wiley \& Sons, Inc., 2001.

[3] T. Kronhamn, "Bearings-only target motion analysis based on a multihypothesis Kalman filter and adaptive ownship motion control," IEE Proceedings - Radar, Sonar and Navigation, vol. 145, no. 4, pp. $247-252$, Aug. 1998.

[4] J. M. C. Clark, R. B. Vinter, and M. M. Yaqoob, "Shifted Rayleigh filter: a new algorithm for bearings-only tracking," IEEE Trans. on Aerospace and Electronic Systems, vol. 43, no. 4, pp. 1373-1384, Oct. 2007.

[5] S. Julier, J. Uhlmann, and H. F. Durrant-Whyte, "A new method for the nonlinear transformation of means and covariances in filters and estimators," IEEE Trans. on Automatic Control, vol. 45, no. 3, pp. 477-482, Mar. 2000.

[6] S. Kay, Fundamentals of Statistical Signal Processing, Vol. I - Estimation Theory. Prentice Hall, 1993.

[7] M. S. Arulampalam, S. Maskell, N. Gordon, and T. Clapp, "A tutorial on particle filters for online nonlinear/non-Gaussian Bayesian tracking," IEEE Trans. on Signal Processing, vol. 50, no. 2, pp. 174 188, Feb. 2002

[8] D. Cox, J. Little, and D. O'Shea, Using Algebraic Geometry. Springer, 2005.

[9] G. P. Huang, K. X. Zhou, N. Trawny, and S. I. Roumeliotis, "A bank of MAP estimators for single-sensor range-only target tracking," in Proc. American Control Conference, Baltimore, MD, June30-July2, 2010, pp. 6974-6980.

[10] A. Farina, "Target tracking with bearing-only measurements," Signal Processing, vol. 78, pp. 61-78, Oct. 1999.

[11] V. Aidala and S. Hammel, "Utilization of modified polar coordinates for bearings-only tracking," IEEE Trans. on Automatic Control, vol. 28, no. 3, pp. 283-294, Mar. 1983.

[12] D. Mušicki, "Bearings only single-sensor target tracking using Gaussian mixtures," Automatica, vol. 45, no. 9, pp. 2088-2092, Sept. 2009.

[13] F. Gunnarsson, N. Bergman, U. Forssell, J. Jansson, R. Karlsson, and P.-J. Nordlund, "Particle filters for positioning, navigation and tracking," IEEE Trans. on Signal Processing, vol. 50, no. 2, pp. 425437, Feb. 2002.

[14] B. Ristic, S. Arulampalam, and N. Gordon, Beyond the Kalman Filter: Particle Filters for Tracking Applications. Artech House, 2004.

[15] F. Gustafsson, "Particle filter theory and practice with positioning applications," IEEE Aerospace and Electronic Systems Magazine, vol. 25 , no. 7 , pp. $53-82$, July 2010 .

[16] G. P. Huang and S. I. Roumeliotis, "Bearing-only tracking using bank of MAP estimators," MARS Lab, University of Minnesota, Minneapolis, MN, Tech. Rep., Feb. 2010. [Online]. Available: www.cs.umn.edu/ ghuang/paper/TR_BOT.pdf

[17] B. Triggs, P. McLauchlan, R. Hartley, and Fitzgibbon, "Bundle adjustment - a modern synthesis," in Vision Algorithms: Theory and Practice. Springer Verlag, 2000, pp. 298-375.

[18] I. Z. Emiris and A. Rege, "Monomial bases and polynomial system solving," in Proc. of the International Symposium on Symbolic and Algebraic Computation, Oxford, UK, July 20-22, 1994, pp. 114-122.

[19] G. H. Golub and C. F. V. Loan, Matrix Computations. The Johns Hopkins University Press, 1996.

[20] H. Stark and J. W. Woods, Probability and Random Processes with Applications to Signal Processing. Prentice Hall, 2001.

[21] A. H. Jazwinski, Stochastic Processes and Filtering Theory. Academic Press, 1970

[22] A. Edelman and H. Murakami, "Polynomial roots from companion matrix eigenvalues," Mathematics of Computation, vol. 64, no. 210, pp. 763-776, Apr. 1995

[23] D. P. Bertsekas, Nonlinear Programming. Athena Scientific, 1999.

[24] Y. Jabri, The Mountain Pass Theorem: Variants, Generalizations and Some Applications. Cambridge University Press, 2003.

[25] A. Doucet, N. de Freitas, and N. Gordon, Eds., Sequential Monte Carlo Methods in Practice. Springer, 2001.

[26] K. Zhou and S. I. Roumeliotis, "Optimal motion strategies for range-only constrained multisensor target tracking," IEEE Trans. on Robotics, vol. 24, no. 5, pp. $1168-1185$, Oct. 2008. 